\title{
Ultraviolet-excited radiative decay channels of defect states in high-density sixfold-coordinated $\mathrm{SiO}_{2}$
}

\author{
A. Paleari, ${ }^{*}$ N. Chiodini, D. Di Martino, and F. Meinardi \\ Istituto Nazionale Fisica della Materia-Dipartimento di Scienza dei Materiali, Università di Milano-Bicocca, \\ via Cozzi 53, I-20125 Milano, Italy \\ P. Fumagalli \\ Dipartimento di Scienze della Terra, Università degli Studi di Milano, via Botticelli 23, I-20133 Milano, Italy
}

(Received 25 June 2003; revised manuscript received 22 July 2003; published 18 November 2003)

\begin{abstract}
Optical emissions at 2.1, 2.6, 3.0, and $4.5 \mathrm{eV}$, excited by synchrotron radiation from 5 to $18 \mathrm{eV}$, were found in stishovite, a high-density sixfold-coordinated $\mathrm{SiO}_{2}$ polymorph. Luminescence and lifetime data were analyzed from 10 to $300 \mathrm{~K}$ showing a transition scheme distinct from that of silica, despite some similarities in the spectral positions. The results give a basis for the study of defect structures in sixfold-coordinated $\mathrm{SiO}_{2}$ environments, for theoretical calculations of the optical response of high-density $\mathrm{SiO}_{2}$, and for a microfluorescence identification of geologic stishovite.
\end{abstract}

DOI: 10.1103/PhysRevB.68.184107

PACS number(s): 78.55.Hx, 61.72.Ji, 81.40.Vw, 91.60.Mk

\section{INTRODUCTION}

Stishovite is peculiar among the natural polymorphs of silicon dioxide for its sixfold-coordinated bonding structure. Other oxides of IV-group atoms show sixfold coordination: it is the stable bonding structure of $\mathrm{SnO}_{2}$ in the cassiterite form and in rutile $\mathrm{GeO}_{2}$. By contrast, the typical bonding structure of silicon dioxide is tetrahedral, prototypes being quartz and silica. Sixfold coordination is not easily sustained in $\mathrm{SiO}_{2}$ and high pressure is required to stabilize the stishovite structure $^{1,2}$ as well as other related high-density phases recently synthesized. ${ }^{1-3}$ Indeed, natural stishovite is mainly found in meteor impact craters but, after the discovery of stishovite by Stishov and Popova, ${ }^{4}$ several studies were carried out on the synthesis and phase stability of high-pressure forms of $\mathrm{SiO}_{2}$ for the important implications in geophysics ${ }^{5}$ as possible $\mathrm{SiO}_{2}$-free phase in the earth's mantle. Nevertheless, other scientific and technological areas are potentially interested in high-pressure polymorphs of $\mathrm{SiO}_{2}$ as model systems where the silicon coordination is far from the more stable tetrahedral bonding structure of silica: deviations from the average fourfold coordination, mainly as point defects, play an important role in many optical applications of silica, and the attribution of the optical extrinsic features due to optically active point defects are currently being debated. ${ }^{6,7}$ This field of investigation was becoming more and more important in the last decade with the discovery of an increasing number of possible applications of silica-based materials, ${ }^{8-10}$ in photorefractive writing, Raman amplification, vacuum ultraviolet (UV) photolithography, nonlinear optical applications, and quantum dots in glass. In these perspectives, the knowledge of the optical features of highdensity polymorphs of silicon dioxide may play a key role in understanding physical processes involving silica coordination defects. Indeed, although no sixfold-coordinated amorphous form of covalent oxides is known, consistent with the continuous random network model of a glass, ${ }^{11,12}$ point defects belonging to overcoordinated complexes might in prin- ciple occur in silica as locally stabilized $\mathrm{SiO}_{2}$ polymorphs. Sixfold coordinated complexes have been recently proposed to occur in amorphous $\mathrm{SiO}_{2}$ (Ref. 13) on the basis of similarities between the photoluminescences (PL) of silica and the cathodoluminescence in rutilelike $\mathrm{GeO}_{2}$ and $\mathrm{SiO}_{2} \cdot{ }^{13-15}$ This conjecture is alternative to more generally agreed attributions of the vacuum-UV excited silica PL to oxygendeficient point defects in the tetrahedrally coordinated $\mathrm{SiO}_{2}$ network. ${ }^{16-18}$ However, no exhaustive experimental data on the optical properties of stishovite near its energy-band gap has yet been collected, to our knowledge. Aim of the present work is to identify the subband gap optical activity of stishovite in the excitation region near and above the energy gap. As a result, a direct experimental basis is given to discuss the role of sixfold-coordinated silicon in the stabilization of high-energy excitation channels of point defects in silica as well as to verify $a b$ initio calculations ${ }^{19-24}$ of the intrinsic properties of the prototype of high-density $\mathrm{SiO}_{2}$ phases.

\section{EXPERIMENT}

High-pressure synthesis of stishovite was carried out starting from quartz powder and applying a pressure of 9 $\mathrm{GPa}$ at $1170 \mathrm{~K}$ in a Walker-type multianvil apparatus ${ }^{25} \mathrm{em}$ ploying tungsten carbide cubes of 32-mm edge length. Pressure cells were made of $\mathrm{MgO}$ octahedra (containing $5 \mathrm{wt} \%$ of $\mathrm{Cr}_{2} \mathrm{O}_{3}$ ) with a 25-mm edge length. Assemblies were composed of a straight graphite heater, $\mathrm{MgO}$ spacers, and axial thermocouple (Pt-Pt90Rh10, $S$ type) insulated by a mullite ceramic and placed in direct contact with the Pt capsule. Graphite discs on both ends of the assembly provided electrical contact with the WC cubes. Pressure calibration was performed both at room temperature and $1273 \mathrm{~K}$, using phase transitions of known materials. Final accuracy of pressure was estimated to be $\pm 4 \%$. Temperature uncertainty at high temperature was $\pm 20 \mathrm{~K}$. Final powder samples were first characterized by X-ray powder diffraction on a Philips APD 1000 diffractometer and by Raman analysis, by means of a spectrometer (Labram Dilors) with excitation at 488 and 


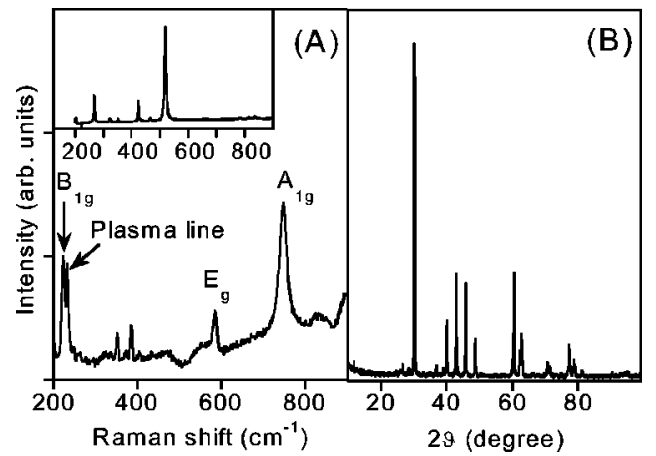

FIG. 1. (a) Raman spectrum (plasma line) and (b) x-ray diffraction of the synthetic stishovite powder samples. Inset: Raman spectrum of a coesite reference sample.

$633 \mathrm{~nm}$ and resolution of $1 \mathrm{~cm}^{-1}$. A reference sample of coesite was also synthesized at lower pressure. PL measurements were carried out by employing synchrotron radiation at the SUPERLUMI experimental station of HASYLAB at DESY (Hamburg, Germany), in the temperature range between 9 and $300 \mathrm{~K}$. The excitation spectral bandwidth was $0.3 \mathrm{~nm}$. Photoluminescence excitation (PLE) and PL signals were detected by a charge-coupled device camera with $3 \mathrm{~nm}$ of emission bandwidth. Data were corrected for the spectral dependence of the excitation intensity. Time-resolved measurements in the nanosecond domain were carried out with a time window of $300 \mathrm{~ns}$ and $0.8 \mathrm{~ns}$ of excitation pulse duration. Slow PL lifetimes were measured by chopping the excitation with a time window of 80 or $160 \mu \mathrm{s}$ and 5 or $10 \mu \mathrm{s}$ of pulse rise time, respectively.

\section{RESULTS}

Figure 1(a) shows the Raman-scattering spectrum of the investigated sample, showing the expected $B_{1 g}, E_{g}$, and $A_{1 g}$ vibrational modes at about 230,590, and $750 \mathrm{~cm}^{-1}$ [26]. No other $\mathrm{SiO}_{2}$ phase is detectable, specifically coesite which should give an intense narrow peak at $520 \mathrm{~cm}^{-1}$ (see inset). Confirmation of a substantially complete phase transformation in stishovite comes from x-ray diffraction (XRD) data shown in Fig. 1(b), all reflections corresponding to the stishovite diffraction pattern. ${ }^{4}$

The spectral distribution of PL intensity as a function of

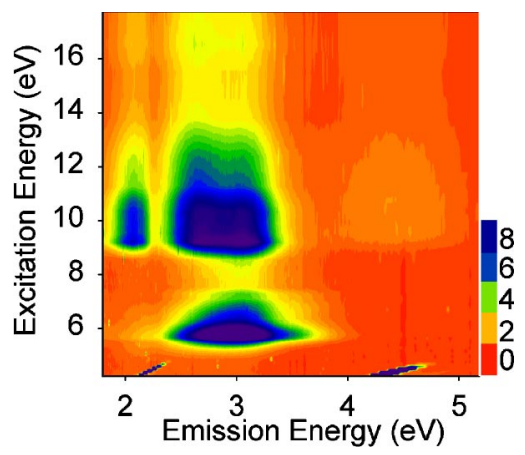

FIG. 2. (Color online) 3D plot of emission-excitation PL intensity pattern of stishovite at $190 \mathrm{~K}$.

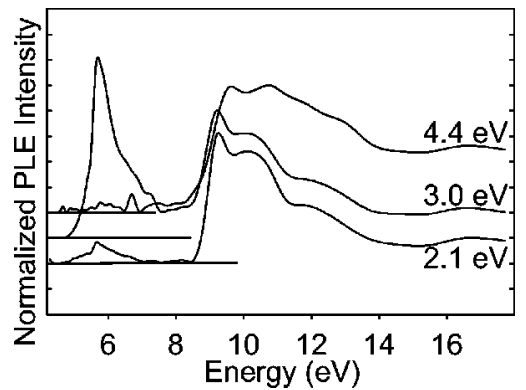

FIG. 3. Excitation spectra at $190 \mathrm{~K}$ at the indicated emission energies in stishovite. Data are normalized and vertically shifted for clarity.

the excitation energy is summarized by the threedimensional (3D) plot in Fig. 2. Emissions are observed at $2.1,2.6,3.0$, and $4.5 \mathrm{eV}$. The excitation pattern shows two main structures at 6-7 and 9-14 eV. The low-energy excitation structure pertains to the emissions at 2.6 and $3 \mathrm{eV}$ only. Instead, the higher-energy portion of the excitation pattern is common to all emissions, although with some differences (see Fig. 3),

Time-resolved PL measurements show two distinct sets of lifetime $\tau_{P L}$ values (summarized in Table I): fast radiative decay processes characterize the PL bands centered at 2.1, 2.6, and $3.0 \mathrm{eV}$, with lifetime of few nanoseconds, when excited in the extended high-energy excitation region above $9 \mathrm{eV}$. The UV emission at $4.5 \mathrm{eV}$ has instead a very slow decay, the lifetime being about $90 \mu \mathrm{s}$. Excitation below $8 \mathrm{eV}$ gives rise to slow emission decays of the PL bands at 2.6 and $3.0 \mathrm{eV}$.

The kind of excitation channel, at low or high energy, also influences the temperature dependence of PL intensity. Figure 4 shows PL spectra for two representative excitation energies with their respective temperature dependence. In the low excitation energy region, the radiative decay is thermally assisted, with a maximum at $200 \mathrm{~K}$, whereas it decreases by increasing the temperature by exciting above $9 \mathrm{eV}$.

\section{DISCUSSION}

The origin of the UV emission at $4.5 \mathrm{eV}$ appears distinct from the low-energy PL bands, either as excitation spectrum or emission lifetime. Its spectral position, not so far from the onset of interband excitations, suggests an attribution to band-to-band recombination. The long lifetime would be

TABLE I. PL lifetime values $\tau_{P L}$ at different emission and excitation energies ( $E_{e m i}$ and $E_{e x c}$, respectively) at $10 \mathrm{~K}$. Uncertainty is about $10 \%$.

\begin{tabular}{ccc}
\hline \hline$E_{\text {emi }}(\mathrm{eV})$ & $E_{\text {exc }}(\mathrm{eV})$ & $\tau_{P L}(\mathrm{~ns})$ \\
\hline 4.5 & 10.3 & $9 \times 10^{4}$ \\
3.1 & 10.3 & 5 \\
2.6 & 10.3 & 7 \\
3.0 & 6.9 & $2 \times 10^{4}$ \\
3.0 & 5.4 & $2 \times 10^{4}$ \\
\hline \hline
\end{tabular}




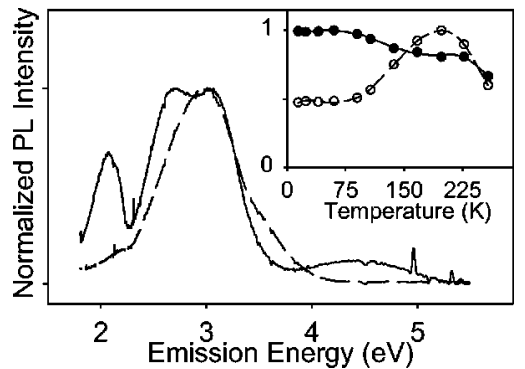

FIG. 4. Emission spectra at $190 \mathrm{~K}$ excited at $10.3 \mathrm{eV}$ (continuous line) and $7 \mathrm{eV}$ (dashed line). Inset: temperature dependence of normalized PL intensity from 10 to $300 \mathrm{~K}$ at $3 \mathrm{eV}$ excited at 10.3 $\mathrm{eV}$ (filled marks) and $7 \mathrm{eV}$ (open marks).

consistent with a low recombination rate caused by the particular symmetries of the wave functions at the edges of the valence and conduction bands. ${ }^{20,21}$

By contrast, the thermally activated PL yield of the emissions at 2.1,2.6, and $3 \mathrm{eV}$ appears consistent with an attribution to defect states. The presence of a relatively narrow excitation peak at $9.3 \mathrm{eV}$ at the onset of the band-to-band excitations, accompanied by a large Stokes shift, would also suggest a possible involvement of these emissions in decay channels from exciton self-trapping. The excitonic peak of quartz is indeed observed at $10.3 \mathrm{eV}$ (Ref. 21) and gives rise to radiative decay at $2.7 \mathrm{eV} .{ }^{13}$ Following this analogy, stishovite appears to give rise to a narrower optical gap with respect to the tetrahedral quartz structure, in qualitative agreement with calculations. ${ }^{21}$

The available data do not allow us to associate all the low-energy emissions to a single kind of defect. In fact, the emission at $2.1 \mathrm{eV}$ does not show the low-energy excitation at $6-8 \mathrm{eV}$, observed for the 2.6 and $3 \mathrm{eV}$ emissions, suggesting a somehow different origin. The agreement between excitation spectra of the 2.6 and $3 \mathrm{eV}$ emissions instead suggests an assignment to a single kind of defect. Figure 5 summarizes a possible scheme of the involved transitions, consistent with the present data. Further details of the involved transitions can be obtained by considering the large change of PL lifetime these emissions show, depending on the excitation. Within a single-defect description, this feature cannot be ascribed to a change of radiative transition rate $k_{R}=\tau_{R}^{-1}$, the transition probability being related to the same

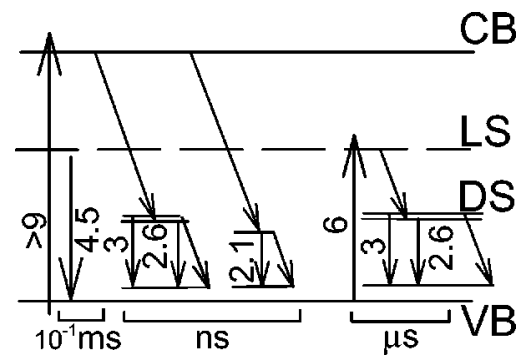

FIG. 5. Scheme of optical transitions (with energy values in eV) involving conduction and valence bands (CB, VB), localized states (LS), and defect states (DS) in stishovite. Diagonal arrows represent nonradiative decays. Time domains of PL lifetime are also indicated. initial and final states in each emission, independently of the excitation path. Therefore, a change of quantum yield $\eta$ has to be considered, caused by a largely different contribution of nonradiative processes with rate $k_{n R}$. These processes are less efficient exciting below $8 \mathrm{eV}$, long lifetimes being observed in this case. Taking into account that

$$
\eta=\frac{\tau_{P L}}{\tau_{R}}=\frac{k_{R}}{k_{R}+k_{n R}},
$$

we can estimate a lower limit for $\tau_{R}$ of the order of $10^{-5} \mathrm{~s}$, typical of a substantially forbidden transition. Excitation below $8 \mathrm{eV}$ is also correlated with a PL yield that increases with the temperature. If the low-energy excitation at $6-7 \mathrm{eV}$ is an intradefect process, a potential barrier between excited defect states may be responsible for this feature, similar to twofold-coordinated (TC) Si sites in silica. ${ }^{6}$ However, the low-energy excitation region might also arise from electron excitations towards localized states in the low-energy tail of the conduction band, with a thermal activation for the migration and/or trapping in defect sites through hopping mechanisms.

By contrast, when the 2.6 and $3 \mathrm{eV}$ emissions are excited in the interband transition region, short PL lifetimes are observed, and a thermal activation of the PL yield is not observed any more. In this case, decay channels with trapping and recombination of electron-hole pairs in defect sites are involved with a great amount of energy excess to be dissipated, the Stokes shift being more than $5 \mathrm{eV}$. As a consequence, nonradiative decay are greatly favored. The effect on $\eta$ is described by the expression

$$
\eta=\frac{k_{R}}{k_{R}+k_{n R}^{0} e^{-E / k T_{e f f}}},
$$

where $T_{\text {eff }}$ is an effective temperature, dependent on the excitation path, which accounts for phonon excitations within the excited defect state during the localization of the excitation. Specifically, $T_{\text {eff }}$ may be very large when a defect site localizes band-to-band excitations. In this case, the denominator in Eq. (2) is dominated by the nonradiative $k_{n R}$ rate, $k_{R}$ being relatively small, as discussed above. The comparison between $\tau_{P L}$ in the two excitation regions shows that $k_{n R}$ $\sim 10^{3} k_{R}$. Such a large nonradiative rate would also explain why interband excitations above $9 \mathrm{eV}$ do not result in a much more intense defect-related PL than that excited at low energy.

The collected data do not enable the identification of specific defect configurations. However, some comments can be made on a possible role of silicalike defects: the sixfoldcoordinated structure of stishovite might contain undercoordinated silicon and oxygen sites such as TC Si sites, unpaired electron in threefold-coordinated $\mathrm{Si}$ sites ( $E^{\prime}$ centers) and nonbridging oxygen (NBO). Possible $E^{\prime}$-like centers were indeed identified by Devine ${ }^{27}$ in irradiated stishovite, and PL from TC Ge sites was observed in sixfold-coordinated rutile $\mathrm{GeO}_{2}$. The spectral position of the emissions at 4.5, 2.6-3, and $2.1 \mathrm{eV}$ might apparently recall some known PL bands in silica, ${ }^{6}$ specifically the singlet-to-singlet $4.2 \mathrm{eV}$ and triplet- 
to-singlet $2.7 \mathrm{eV}$ (3.1 $\mathrm{eV}$ in fused natural quartz) emissions of TC sites, and the NBO PL band at $1.9 \mathrm{eV}$. However, several important differences should be considered: (i) the emissions at 4.5 and $2.6-3 \mathrm{eV}$ in stishovite show distinct excitation spectra, whereas a single excitation pattern pertains to the 4.2 and 2.7 (3.1) eV emissions of silica; (ii) the $4.5-\mathrm{eV}$ emission in stishovite has a long lifetime, a factor $10^{4}$ slower than the lifetime of the 2.6-3 eV PL at the same excitation energy (or a factor 10 with respect to the $2.6-3 \mathrm{eV}$ bands when excited below $8 \mathrm{eV}$ ), whereas the opposite occurs in silica where the 4.2-eV PL is much faster than the $2.7-3 \mathrm{eV}$ emissions; (iii) the temperature dependence of PL intensity at $2.6-3 \mathrm{eV}$ in stishovite is the same of that at $4.5 \mathrm{eV}$ by exciting above $9 \mathrm{eV}$ and it changes by exciting below $8 \mathrm{eV}$ where the $4.5 \mathrm{eV}$ band is not observed any more, whereas in silica the 4.2 and $2.7(3.1) \mathrm{eV}$ bands are strictly intensity anticorrelated; (iv) the emission at $2.1 \mathrm{eV}$ does not show low-energy excitation (also verified in the red by HeNe laser excitation), whereas a quasiresonant excitation at $2.1 \mathrm{eV}$ of the red $1.9 \mathrm{eV}$ PL of NBO is observed in silica. As a result of this analysis, blue-green emissions of silica, no matter where they are excited and despite some apparent spectral similarities with emissions of stishovite, cannot be straightforwardly identified with the optical activity of defects in a sixfold coordinated $\mathrm{SiO}_{2}$ network. The peculiar bonding structure of stishovite might be responsible for the observed differences.
However, different defect configurations should also be considered, as well as interface defects and dislocations possibly occurring in our powder polycrystalline sample.

\section{SUMMARY}

We have obtained spectroscopical and lifetime data on the UV-excited PL of stishovite showing clear-cut differences with respect to the known phenomenology of silica. As a result, a basis is given to ground theoretical studies on the optical properties of possible silicalike or other type of defects in stishovite. Furthermore, the picture gives indication of the intrinsic properties of stishovite, suggesting an optical gap narrower than that in silica, in qualitative agreement with theoretical calculations, but about $2-3 \mathrm{eV}$ above the theoretical value, consistent with an expected but previously undefined underestimation.

\section{ACKNOWLEDGMENTS}

The authors thank Stefano Poli for useful discussions. This work was part of a National Project partially supported by the Italian Government. Experiment at HASYLAB was supported by the IHP-Contract No. HPRI-CT-1999-00040/ 2001-00140 of the European Commission.
*Electronic address: alberto.paleari@mater.unimib.it

${ }^{1}$ M. Murakami, K. Hirose, S. Ono, and Y. Ohishi, Geophys. Res. Lett. 30, 1207 (2003).

${ }^{2}$ S. Ono, K. Hirose, M. Murakami, and M. Isshiki, Earth Planet. Sci. Lett. 197, 187 (2002).

${ }^{3}$ L.S. Dubrovinsky, S.K. Saxena, P. Lazor, R. Ahuja, O. Eriksson, J.M. Wills, and B. Johansson, Nature (London) 388, 362 (1997).

${ }^{4}$ M. Stishov and S.V. Popova, Geokhimiya 10, 837 (1961).

${ }^{5}$ K.J. Kingma, R.E. Cohen, R.J. Hemley, and H.K. Mao, Nature (London) 374, 243 (1995).

${ }^{6}$ L. Skuja, in Defects in $\mathrm{SiO}_{2}$ and Related Dielectrics: Science and Technology, edited by G. Pacchioni, L. Skuja, and D. L. Griscom (Kluwer, Dordrecht, MA, 2000), p. 73.

${ }^{7}$ D.L. Griscom, J. Ceram. Soc. Jpn. 99, 899 (1991).

${ }^{8}$ J. Albert, MRS Bull. 23, 36 (1998).

${ }^{9}$ M.J. Dejneka, MRS Bull. 23, 57 (1998).

${ }^{10}$ W. Margulis, F.C. Garcia, E.N. Hering, L.C. Guedes Valente, B. Lesche, F. Laurell, and I.C.S. Carvalho, MRS Bull. 23, 31 (1998).

${ }^{11}$ W.H. Zachariasen, J. Am. Chem. Soc. 54, 3841 (1932).

${ }^{12}$ J.C. Phillips, J. Non-Cryst. Solids 34, 153 (1979).
${ }^{13}$ H.J. Fitting, T. Barfels, A.N. Trukhin, and B. Schmidt, J. NonCryst. Solids 279, 51 (2000).

${ }^{14}$ A.N. Trukhin and P.A. Kulis, J. Non-Cryst. Solids 188, 125 (1995).

${ }^{15}$ A.N. Trukhin, J. Non-Cryst. Solids 189, 291 (1995).

${ }^{16}$ L. Skuja, J. Non-Cryst. Solids 239, 16 (1998).

${ }^{17}$ G. Pacchioni and G. Ierano, Phys. Rev. Lett. 79, 753 (1997).

${ }^{18}$ D. Donadio, M. Bernasconi, and M. Boero, Phys. Rev. Lett. 87, 195504 (2001).

${ }^{19}$ D.D. Klug, R. Rousseau, K. Uehara, M. Bernasconi, Y.L. Page, and J.S. Tse, Phys. Rev. B 63, 104106 (2001).

${ }^{20}$ J.K. Rudra and W.B. Fowler, Phys. Rev. B 28, 1061 (1983).

${ }^{21}$ Yong-nian Xu and W.Y. Ching, Phys. Rev. B 44, 11048 (1991).

${ }^{22}$ R.E. Cohen, Am. Mineral. 76, 733 (1991).

${ }^{23}$ Y.P. Li and W.Y. Ching, Phys. Rev. B 31, 2172 (1985).

${ }^{24}$ J.R. Alvarez and P. Rez, Solid State Commun. 108, 37 (1998).

${ }^{25}$ P. Fumagalli and S. Poli, Periodico di Mineralogia 68, 275 (1999).

${ }^{26}$ R.J. Hemley, H. Mao, P.M. Bell, and S. Akimoto, Physica B 139/ 140, 455 (1986).

${ }^{27}$ R.A.B. Devine and K. Hübner, Phys. Rev. B 40, 7281 (1989). 